\title{
Zinc supplementation for treating diarrhea in children: a systematic review and meta-analysis
}

\author{
Tais Freire Galvao, ${ }^{1}$ Maria Fernanda Reis e Silva Thees, ${ }^{2}$ \\ Rossana Ferreira Pontes, ${ }^{3}$ Marcus Tolentino Silva, ${ }^{1}$ \\ and Mauricio Gomes Pereira ${ }^{4}$
}

Suggested citation

Galvao TF, Thees MFRS, Pontes RF, Silva MT, Pereira MG. Zinc supplementation for treating diarrhea in children: a systematic review and meta-analysis. Rev Panam Salud Publica. 2013;33(5):370-7.

ABSTRACT Objective. To update the available evidence about zinc use for treating diarrhea in children and to assess its effect on the malnourished population, a subgroup that has not been fully explored in previous analyses.

Methods. A systematic review was performed of randomized clinical trials that assessed children up to 5 years old with acute diarrhea who received zinc supplementation. Controls received a placebo or oral rehydration therapy. After searching the main databases, without language restrictions, two independent reviewers selected eligible studies, extracted the data, and assessed the risk of bias of included studies. Meta-analyses were calculated using MantelHaenszel or inverse variance random effects.

Results. Eighteen of 1041 studies retrieved were included in the review ( $\mathrm{n}=7314$ children). Zinc was beneficial for reducing the duration of diarrhea in hours (mean difference $[M D]=-20.12,95 \%$ confidence interval $[C I]=-29.15$ to $\left.-11.09, \mathrm{I}^{2}=91 \%\right)$. The effect was greater in malnourished children $\left(M D=-33.17,95 \% C I=-33.55\right.$ to $\left.-27.79, \mathrm{I}^{2}=0 \%\right)$. Diarrhea prevalence on days 3, 5, and 7 was lower in the zinc group. The incidence of vomiting was significantly greater in the group that received zinc than in the control group. Included randomized controlled trials were of low risk of bias in most domains assessed.

Conclusions. Oral zinc supplementation significantly decreases diarrhea duration and has a greater effect on malnourished children. Zinc supplementation seems to be an appropriate public health strategy, mainly in areas of endemic deficiencies.

Key words Zinc; diarrhea; child; malnutrition; review.

Diarrhea is responsible for $15 \%$ of all deaths in children under 5 years of age and accounts for about 1.4 million

\footnotetext{
${ }^{1}$ Faculdade of Medicina, Universidade de Brasilia, Brasilia, Brazil. Send correspondence to: Tais Freire Galvao, taisgalvao@gmail.com

2 Setor de Indústria e Abastecimento, Agência Nacional de Vigilância Sanitária, Brasilia, Brazil.

${ }^{3}$ Setor de Areas Isoladas Norte, Secretaria de Saúde do Distrito Federal, Brasilia, Brazil.

4 Programa de Pós-Graduação em Ciências da Saúde, Faculdade de Ciências da Saúde, Universidade de Brasília, Brasilia, Brazil.
}

annual infant deaths worldwide $(1,2)$. Data from the World Health Organization (WHO) and the United Nations Children's Fund (UNICEF) have shown a substantial decrease in deaths in adults over the age of 30 , and the mortality rate for children under age 5 from acute diarrhea has fallen from 4.5 million deaths annually in 1979 to 1.6 million deaths in 2002. Despite these improvements, mortality due to diarrhea in developing countries remains too high (3).
Severe diarrhea can quickly cause life-threatening dehydration. One major advance in reducing mortality from diarrhea was the introduction of $\mathrm{WHO}$ oral rehydration solution (ORS). However, WHO ORS is unable to reduce the volume, frequency, and duration of diarrhea; consequently, other approaches to improve or supplement ORS have been researched (3). Among the available treatments, WHO currently recommends early oral rehydration therapy and zinc 
supplementation to treat diarrhea in children between 6 and 60 months of age (1, 4-6). This recommendation is based on systematic reviews (7-11) that have demonstrated the beneficial effect of zinc treatment in reducing the duration and severity of diarrhea episodes in children less than 5 years old $(4,6,12)$. However, the effect of zinc in malnourished children is less known. The aim of this systematic review is to update the available evidence on zinc efficacy for treating acute diarrhea in children and to investigate the effect of zinc supplementation on malnourished children.

\section{MATERIALS AND METHODS}

\section{Study eligibility criteria}

Randomized controlled trials (RCTs) that met the following criteria were considered to be eligible for the systematic review: hospital or community based, included children up to 5 years old with acute diarrhea, used zinc sulfate or gluconate associated or not with oral rehydration therapy in any dose for up to 14 days, and used placebo or oral rehydration therapy controls.

Study outcomes included diarrhea duration, stool frequency, and adverse events. Acute diarrhea was defined as three or more episodes of liquid stool within a 24-hour period (7).

The exclusion criteria were as follows: studies that included children with persistent diarrhea, studies that assessed zinc without a defined dose, and studies that assessed zinc as supplementation for prevention of diarrhea and other diseases.

A malnourished child was defined as having a low weight-for-height $Z$ score $(<-2)(13-15)$ or with zinc depletion (below $14 \mu \mathrm{mol} / \mathrm{L}$ ). Other comorbid conditions were not considered, as the study population was heterogeneous and could have various conditions causing low weight.

\section{Data sources and search strategy}

A search was conducted regardless of language or publication status on Medline, Embase, Central, mCRT, LILACS, and SciELO databases. The most recent literature search was performed in January 2013.

The search strategy in Medline (via PubMed) was:
1. "infant"[mesh] or "infants" [tiab] or "child, preschool" [mesh] or "preschool child"[tiab] or "child"[mesh] or "child" [tiab]

2. "diarrhea"[mesh] or "diarrhea"[tiab] or "diarrhea" [tiab]

3. "zinc" [mesh] or "zinc" [tiab]

4. (therapy/broad[filter])

5. (1 and 2 and 3 and 4 ).

\section{Study selection and data extraction}

Two independent reviewers evaluated study eligibility according to titles and abstracts; disagreements were resolved by consensus. Selected studies' full texts were independently assessed for final inclusion.

A form was used to extract relevant data from studies, including study design, country, year, population, setting, blinding, allocation concealment, sample size, intervention, and outcomes. Article references were also searched. Authors were contacted if the full text was not available.

\section{Data analysis and risk of bias}

The primary outcome was mean difference (MD) of diarrhea duration in hours, in addition to $95 \%$ confidence interval (CI). Secondary outcomes were presented according to the type of variable and corresponding 95\% CI: continuous data were presented as mean and standard deviation and summarized by MD; for dichotomous variables, the relative risks and number needed to treat were calculated.

Review Manager 5.1.2 software was used to summarize the results by Mantel-Haenszel or inverse variance random effects and to calculate statistical heterogeneity $\left(I^{2}\right.$, tau-, and chisquared tests). Sensitivity analysis was performed for the dichotomous outcomes when no data were available for some participants (worst-case/best-case scenarios). Plausible causes of heterogeneity were explored: study setting (hospital vs. community), country (low risk of zinc deficiency vs. high risk of zinc deficiency), nutritional status (nourished vs. malnourished), and dose of elemental zinc (> $20 \mathrm{mg}$ vs. $\leq 20 \mathrm{mg}$ ).

The reviewers assessed the risk of bias in the studies using the Cochrane handbook criteria (16). Risk of publication bias was verified by funnel plot asymmetry.

\section{RESULTS}

A total of 1041 studies were retrieved (Figure 1) (17-46). After the titles and abstracts were assessed for eligibility, 18 RCTs were included in the review ( $n=7314$ children).

Included studies were considered to be of acceptable quality with a low risk of bias. Potential biases detected did not appear to compromise the results obtained. All studies were classified as "unclear" for the possibility of the occurrence of selective reporting of the outcomes, as the reviewers were unable to access the study protocols. Almost 50\% of the studies were classified as unclear for allocation concealment, as they did not inform whether patient allocation remained inaccessible to the teams that were directly involved in the studies (Figure 2).

Funnel plot inspection (data not presented) showed asymmetry of the findings, indicating a possible risk of publication bias as it is possible that small studies that found unfavorable results were not published (47).

Study characteristics are shown in Table 1 (29-46). Only three studies had a population of fewer than 100 patients, and all studies were conducted in countries considered to be "emerging and developing economies"(48).

Most of the studies also presented similar outcomes, although the units of measure or form of analysis often differed, thus precluding inclusion of the complete data in the review. This phenomenon occurred particularly in stool frequency data.

In placebo-controlled studies, oral rehydration therapy was used to replace lost fluid in dehydrated children, whether before or during the zinc treatment.

Diarrhea duration comparing zinc with placebo is presented in Figure 3 $(29,31-34,36-38,40,41,43,45,46)$. Despite significant heterogeneity among included studies, zinc reduced the duration of diarrhea in children. Diarrhea prevalence on days 3,5 , and 7 of the study was also lower in the zinc-treated group (Table 2) $(30,32-35,37,38,42,43)$; on day 7 , the difference was statistically significant. According to these results, it is necessary to treat 22 children with zinc to prevent 1 case of diarrhea on day 7 (number needed to treat $=22 ; 95 \%$ $\mathrm{CI}=14-55)$.

As one study (26) excluded a significant number of children in both study 
FIGURE 1. Search, selection, and inclusion of studies

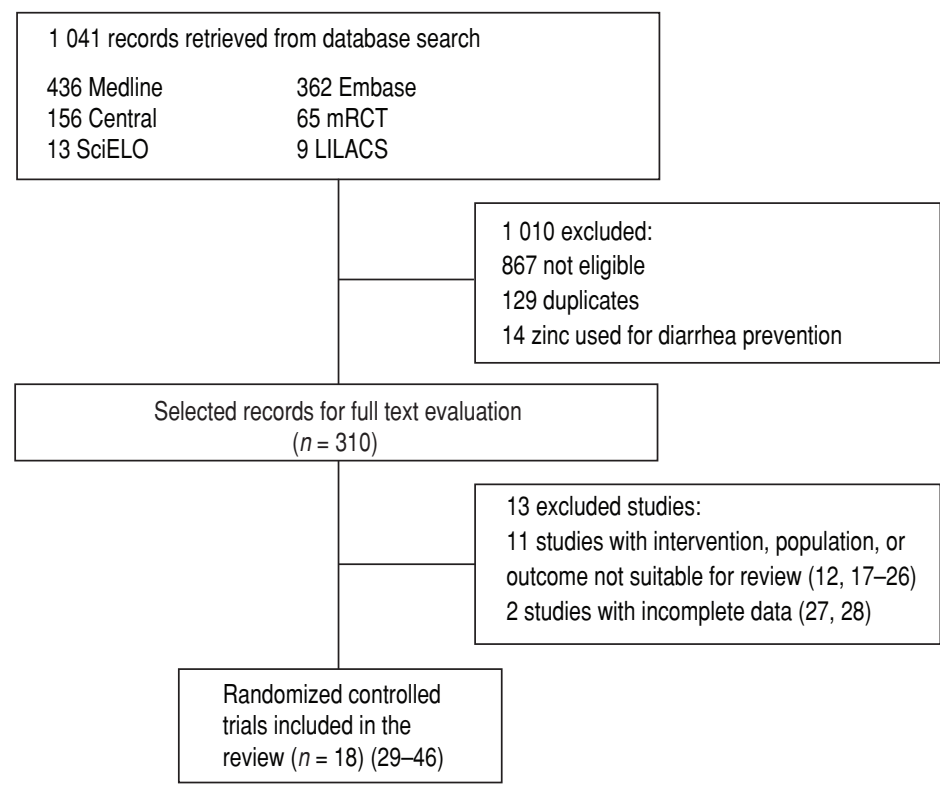

groups, a sensitivity analysis was performed to verify the impact of this exclusion on the outcome reported in the study with regard to the prevalence of diarrhea on day 7. No statistically significant difference was found in the results, even considering that all 16 children excluded from the zinc group and all 24 children excluded from the control group still had diarrhea on day 7 (relative risk $=0.64 ; 95 \% \mathrm{CI}=0.49-0.84$ ).

Four studies evaluated the stool frequency in the mean number of episodes/day $(29,34,36)$, with an average reduction of 2.4 episodes of defecation (95\% CI = 3.79-1.04).

The only relevant adverse reaction reported was vomiting, which had the highest incidence in the zinc-treated group (Figure 4) $(29,30,35,37,39)$.

FIGURE 2. Risk of bias assessment of included studies

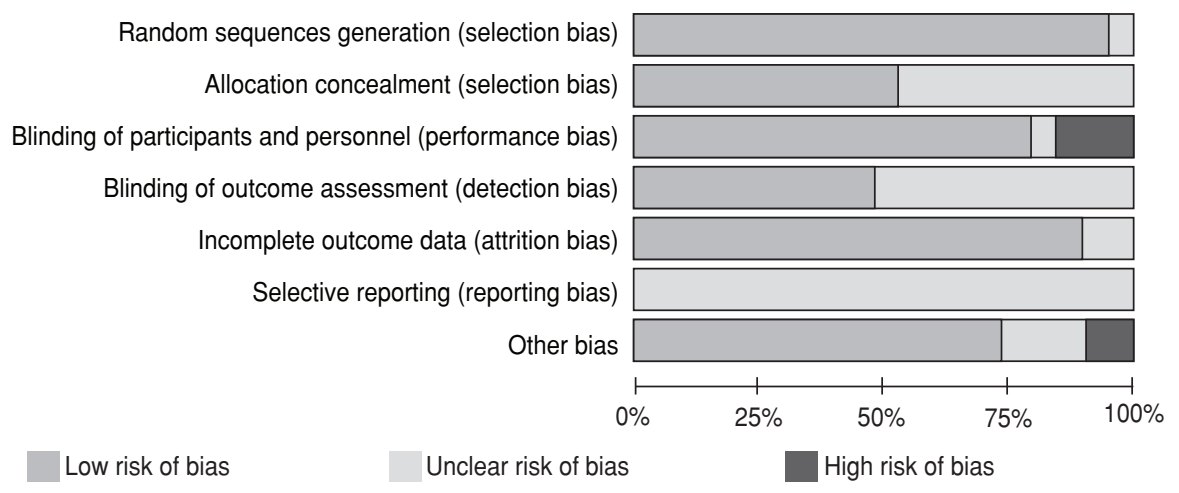

\section{Heterogeneity}

Significant heterogeneity was observed between studies for the outcomes of diarrhea duration and the prevalence of diarrhea on days 3, 5, and 7. The sensitivity analysis showed that the nutritional status of the children might explain the heterogeneity across studies for diarrhea duration. When only malnourished population data were assessed, no heterogeneity was found (Figure 5). As most RCTs did not report separate results for well-nourished and malnourished children, the body of evidence could not be stratified to provide homogeneous results. No other causes of heterogeneity were identified.

The sensitivity analysis also showed that one study (43) contributed considerably toward the heterogeneity detected in the outcomes of diarrhea on days 3, 5 , and 7. This outcome might be due to low adherence to the treatment in the study, which may have contributed to a smaller effect of the use of zinc in the population assessed.

Five studies evaluated only malnourished children or included an evaluation of this subgroup $(31,35,38,41,49)$. Three of these studies $(29,36,37)$ included children with zinc deficiency, who are labeled as malnourished children in this review. Other studies that also included malnourished children were not included in this assessment because a subgroup analysis was not reported in the original papers, thus rendering it impossible to obtain segregated data for this assessment.

The meta-analysis presented in Figure 5 shows zinc as being more effective for this population, as it reduced diarrhea duration by 33.17 hours $(95 \% \mathrm{CI}=-38.55$ to -27.79$)(29,31,33,36,37)$.

\section{DISCUSSION}

This review updates and confirms existing evidence about the efficacy of zinc supplementation in children for reducing diarrhea, with more striking results in malnourished children, which is a population that has not been thoroughly assessed in previous reviews. A significant reduction in diarrhea duration occurred, thus endorsing the findings of previous systematic reviews (7-11).

Zinc is one of the most important micronutrients in the human diet; it is crucial for many cell functions, such as protein synthesis and cell growth and differentiation (49). Zinc's mechanism of action for the treatment of diarrhea caused by different pathogens is not fully understood, but studies conducted in this field reveal that zinc plays different roles in the intestine, such as regulation of intestinal fluid transport and mucosal integrity and modulation of expression of genes encoding important zinc-dependent enzymes like cytokines, which play important roles in the immune system and in modulation of oxidative stress $(49,50)$. These different roles might explain the positive effect of zinc intake during acute diarrhea in children. Zinc supplementation seems 
TABLE 1. Characteristics of included randomized clinical trials

\begin{tabular}{|c|c|c|c|c|c|c|c|c|}
\hline Study & Blinding & $\begin{array}{l}\text { Allocation } \\
\text { concealment }\end{array}$ & $N^{a}$ & Interventions & Population & Setting & Country & Outcomes \\
\hline $\begin{array}{l}\text { Sachdev et al. } \\
1988 \text { (29) }\end{array}$ & Unclear & Unclear & 50 & $\begin{array}{l}\text { Elemental zinc, } 40 \mathrm{mg} \\
+\mathrm{ORT}^{\mathrm{b}} ; \text { placebo + } \\
\mathrm{ORT}^{\mathrm{b}}\end{array}$ & $\begin{array}{l}6-18 \text { months, } \\
\text { malnourished and } \\
\text { nourished children with } \\
\text { acute diarrhea }\end{array}$ & Hospital & India & $\begin{array}{l}\text { Diarrhea duration, stool } \\
\text { frequency, duration of } \\
\text { hospitalization, diarrhea on } \\
\text { day } 7\end{array}$ \\
\hline $\begin{array}{l}\text { Sazawal et al. } \\
1995(30)\end{array}$ & Yes & Yes & 947 & $\begin{array}{l}\text { Elemental zinc, } 20 \mathrm{mg}+ \\
\text { MTVc; placebo + MTVc; } \\
\text { liquid preparation }\end{array}$ & $\begin{array}{l}6-35 \text { months, with } \\
\text { acute diarrhea }\end{array}$ & Community & India & $\begin{array}{l}\text { Stool frequency, reduction } \\
\text { in stool frequency, vomiting } \\
\text { frequency, diarrhea on day } 7\end{array}$ \\
\hline $\begin{array}{l}\text { Faruque et al. } \\
1999(32)\end{array}$ & Yes & Unclear & 684 & $\begin{array}{l}\text { Elemental zinc, } 40 \mathrm{mg}+ \\
\text { vitamin } \mathrm{A}\end{array}$ & $\begin{array}{l}6-24 \text { months with } \\
\text { acute diarrhea }\end{array}$ & Hospital & Bangladesh & $\begin{array}{l}\text { Diarrhea duration, diarrhea } \\
\text { on day } 7\end{array}$ \\
\hline $\begin{array}{l}\text { Dutta et al. } \\
2000 \text { (33) }\end{array}$ & Yes & Yes & 80 & $\begin{array}{l}\text { Elemental zinc, } 40 \mathrm{mg}+ \\
\mathrm{ORT}^{\mathrm{b}} ; \text { placebo + } \mathrm{ORT}^{\mathrm{b}}\end{array}$ & $\begin{array}{l}\text { 3-24 months, male, } \\
\text { malnourished, with } \\
\text { acute diarrhea }\end{array}$ & Hospital & India & $\begin{array}{l}\text { Diarrhea duration, diarrhea on } \\
\text { day } 5 \text {, total stool output }\end{array}$ \\
\hline $\begin{array}{l}\text { Strand et al. } \\
2002(35)\end{array}$ & Yes & Yes & 899 & $\begin{array}{l}\text { Zinc gluconate, } 15 \\
\text { mg (infants) or } 30 \\
\text { mg (older children); } \\
\text { placebo }\end{array}$ & $\begin{array}{l}6-35 \text { months, with } \\
\text { less than } 96 \text { hours of } \\
\text { diarrhea }\end{array}$ & Community & Nepal & $\begin{array}{l}\text { Diarrhea on days } 3 \text { and } 7 \text {, } \\
\text { vomiting frequency }\end{array}$ \\
\hline $\begin{array}{l}\text { Al-Sonboli et } \\
\text { al. } 2003 \text { (36) }\end{array}$ & Yes & Unclear & 81 & $\begin{array}{l}\text { Elemental zinc, } 22.5 \mathrm{mg} \\
\text { (3-6 months) or } 45 \mathrm{mg} \\
\text { (7-60 months) per day + } \\
\text { ORT }^{\mathrm{b}} \text {; placebo + ORT }\end{array}$ & $\begin{array}{l}3-60 \text { months, with } \\
\text { acute diarrhea }\end{array}$ & Hospital & Brazil & $\begin{array}{l}\text { Diarrhea duration, stool } \\
\text { frequency }\end{array}$ \\
\hline $\begin{array}{l}\text { Polat et al. } \\
2003 \text { (37) }\end{array}$ & Yes & Unclear & 200 & $\begin{array}{l}\text { Elemental zinc, } 20 \text { mg; } \\
\text { placebo }\end{array}$ & $\begin{array}{l}2-29 \text { months, } \\
\text { malnourished, with } \\
\text { acute diarrhea }\end{array}$ & Community & Turkey & $\begin{array}{l}\text { Diarrhea duration, stool } \\
\text { frequency on days } 2 \text { and } 4 \text {, } \\
\text { diarrhea on days } 3 \text { and } 7 \text {, } \\
\text { vomiting frequency }\end{array}$ \\
\hline $\begin{array}{l}\text { Boran et al. } \\
2006(40)\end{array}$ & Yes & Unclear & 280 & $\begin{array}{l}\text { Elemental zinc, } 15 \mathrm{mg} \\
\text { (6-12 months) or } 30 \mathrm{mg} \\
\text { (13-60 months) per day; } \\
\mathrm{ORT}^{\mathrm{b}}\end{array}$ & $\begin{array}{l}6-60 \text { months, with } \\
\text { acute diarrhea }\end{array}$ & Hospital & Turkey & $\begin{array}{l}\text { Diarrhea duration; stool } \\
\text { frequency on days } 1,2 \text {, and } 3 \text {; } \\
\text { vomiting frequency }\end{array}$ \\
\hline $\begin{array}{l}\text { Gregorio et } \\
\text { al. } 2007 \text { (41) }\end{array}$ & No & Unclear & 117 & $\begin{array}{l}\text { Zinc sulfate, } 20 \mathrm{mg}+ \\
\text { ORT'; ORT }^{\mathrm{b}}\end{array}$ & $\begin{array}{l}2-59 \text { months, with } \\
\text { acute diarrhea }\end{array}$ & Hospital & Turkey & Diarrhea duration \\
\hline $\begin{array}{l}\text { Roy et al. } \\
2008 \text { (42) }\end{array}$ & Yes & Unclear & 56 & $\begin{array}{l}\text { Elemental zinc, } 10 \mathrm{mg}+ \\
\text { MTVc; placebo + MTVc }\end{array}$ & $\begin{array}{l}12-59 \text { months, with } \\
\text { moderate malnutrition } \\
\text { and culture-positive } \\
\text { shigellosis }\end{array}$ & Hospital & Bangladesh & $\begin{array}{l}\text { Diarrhea duration, diarrhea on } \\
\text { day } 7 \text {, death }\end{array}$ \\
\hline $\begin{array}{l}\text { Patel et al. } \\
2009 \text { (43) }\end{array}$ & Yes & Yes & 808 & $\begin{array}{l}\text { Elemental zinc, } 20 \mathrm{mg} \\
\text { (zinc sulfate); elemental } \\
\text { zinc, } 20 \mathrm{mg}+\text { elemental } \\
\text { copper, } 2 \mathrm{mg} \text { (zinc } \\
\text { sulfate + copper); } \\
\text { placebo }\end{array}$ & $\begin{array}{l}6-59 \text { months, with } \\
\text { acute diarrhea }\end{array}$ & Hospital & India & $\begin{array}{l}\text { Diarrhea duration; diarrhea } \\
\text { on days } 3,5 \text {, and } 7 \text {; total stool } \\
\text { output }\end{array}$ \\
\hline $\begin{array}{l}\text { Patro et al. } \\
2010 \text { (44) }\end{array}$ & Yes & Yes & 141 & $\begin{array}{l}\text { Elemental zinc, } 10 \mathrm{mg} \\
\text { ( } 3-5 \text { months) or } 20 \mathrm{mg} \\
\text { (6-48 months) per day; } \\
\text { placebo }\end{array}$ & $\begin{array}{l}3-48 \text { months, with } \\
\text { acute diarrhea }\end{array}$ & Hospital & Poland & $\begin{array}{l}\text { Diarrhea duration; stool } \\
\text { frequency on days } 1,2 \text {, and } \\
3 \text {; vomiting frequency on days } \\
1,2 \text {, and } 3 \text {; use of intravenous } \\
\text { hydration; diarrhea on day } 7 \text {; } \\
\text { side effects }\end{array}$ \\
\hline
\end{tabular}


TABLE 1. Continued

\begin{tabular}{|c|c|c|c|c|c|c|c|c|}
\hline $\begin{array}{l}\text { Dalgic et al. } \\
2011(45)\end{array}$ & No & Yes & 120 & $\begin{array}{l}\text { Elemental zinc, } 6 \mathrm{mg} \\
\text { (<6 months) or } 12 \mathrm{mg} \\
\text { (> } 6 \text { months) }\end{array}$ & $\begin{array}{l}1-28 \text { months, with } \\
\text { rotavirus diarrhea }\end{array}$ & Hospital & Turkey & Diarrhea duration \\
\hline $\begin{array}{l}\text { Dutta et al. } \\
2011 \text { (46) }\end{array}$ & Yes & Yes & 167 & $\begin{array}{l}\text { Elemental zinc, } 20 \mathrm{mg} \\
\text { daily + ORT } \text {; placebo } \\
+\mathrm{ORT}^{\mathrm{b}}\end{array}$ & $\begin{array}{l}\text { 6-24 months, male, } \\
\text { with acute watery } \\
\text { diarrhea }\end{array}$ & Hospital & India & $\begin{array}{l}\text { Diarrhea duration, volume of } \\
\text { diarrhea, consumption of oral } \\
\text { rehydration solution }\end{array}$ \\
\hline
\end{tabular}

a $N$ : total population.

b ORT: oral rehydration therapy.

c MTV: multivitamins.

FIGURE 3. Diarrhea duration (hours) comparing zinc and controla

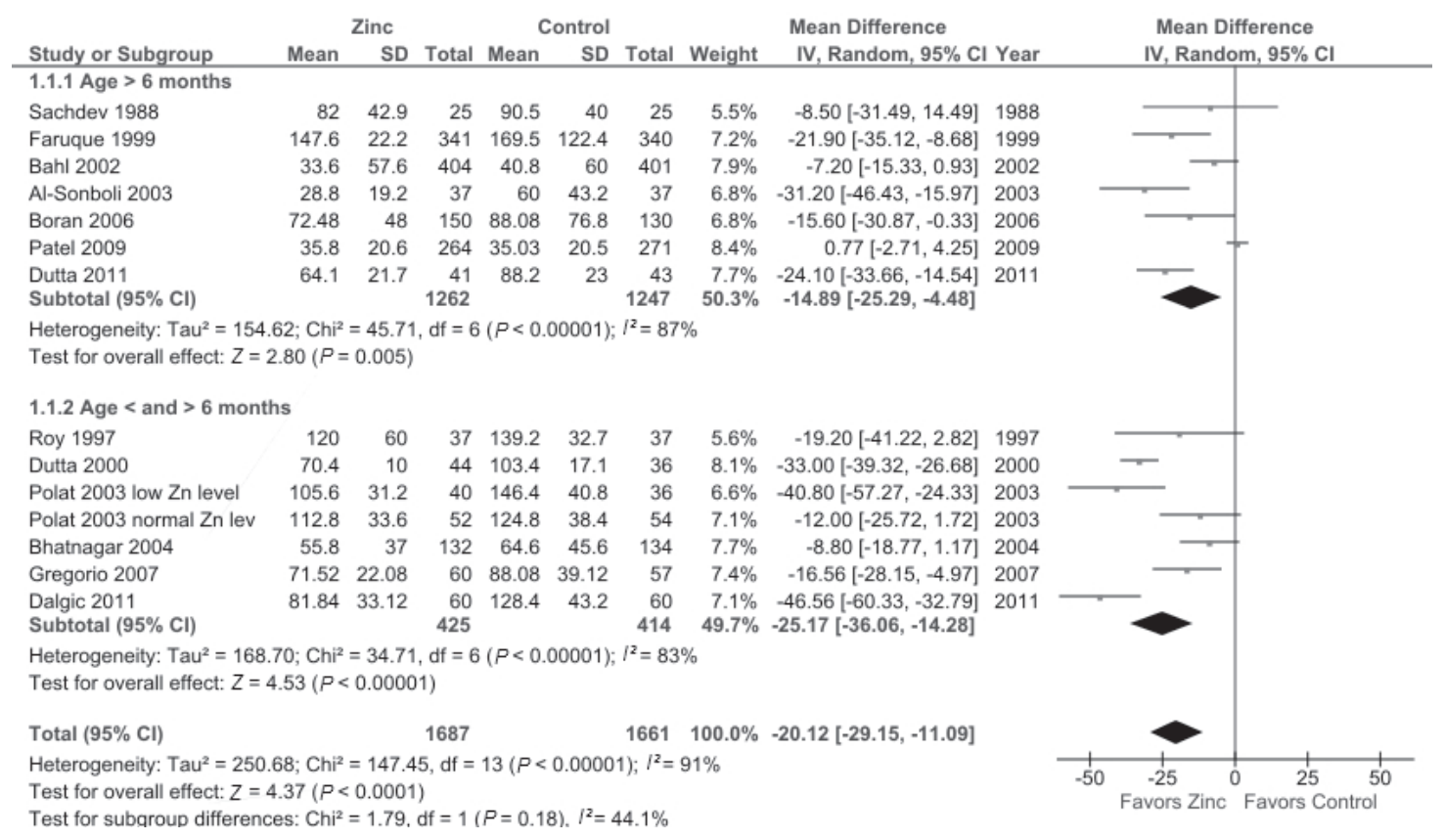

a SD: standard deviation, IV: inverse variance, Cl: confidence interval, df: degrees of freedom.

TABLE 2. Meta-analysis of effect of zinc on prevalence of diarrhea on days 3,5 , and 7

\begin{tabular}{|c|c|c|c|c|c|c|c|c|c|c|}
\hline \multirow[b]{2}{*}{ Study } & \multirow[b]{2}{*}{ Day } & \multicolumn{2}{|c|}{ Zinc } & \multicolumn{2}{|c|}{ Control } & \multirow[b]{2}{*}{ Relative risk } & \multirow[b]{2}{*}{$95 \% \mathrm{Cl}^{\mathrm{C}}$} & \multicolumn{2}{|c|}{ Total effect } & \multirow[b]{2}{*}{ Heterogeneity } \\
\hline & & $n^{a}$ & $N^{\mathrm{b}}$ & $n^{a}$ & $N^{b}$ & & & $Z$ & $P$ & \\
\hline $\begin{array}{l}\text { Bahl et al. } 2002 \text { (34), Strand et al. } 2002 \\
\text { (35), Polat et al. } 2003 \text { (37), Patel } \\
\text { et al. } 2009 \text { (43) }\end{array}$ & 3 & 317 & 1202 & 403 & 1211 & 0.74 & $0.60-0.92$ & 2.69 & 0.007 & $\begin{array}{l}\mathrm{Tau}^{2}=0.04, \text { chi }^{2}=11.52 \\
\mathrm{df}^{\mathrm{d}}=4(P=0.02) \\
I^{2}=65 \%\end{array}$ \\
\hline $\begin{array}{l}\text { Dutta et al. } 2000 \text { (33), Bahl et al. } 2002 \\
\text { (34), Bhatnagar et al. } 2004 \text { (38), } \\
\text { Patel et al. } 2009 \text { (43) }\end{array}$ & 5 & 70 & 844 & 94 & 842 & 0.65 & $0.37-1.12$ & 0.98 & 0.33 & $\begin{array}{l}\mathrm{Tau}^{2}=0.13, \mathrm{chi}^{2}=6.55 \\
\mathrm{df}^{\mathrm{d}}=3(P=0.09) \\
I^{2}=54 \%\end{array}$ \\
\hline $\begin{array}{l}\text { Sazawal et al. } 1995 \text { (30), Faruque et } \\
\text { al. } 1999 \text { (32), Bahl et al. } 2002 \text { (34), } \\
\text { Strand et al. } 2002 \text { (35), Polat et al. } \\
2003 \text { (37), Bhatnagar et al. } 2004 \\
\text { (38), Patel et al. } 2009 \text { (43), Roy } \\
\text { et al. } 2008 \text { (42) }\end{array}$ & 7 & 194 & 2228 & 297 & 2266 & 0.62 & $0.47-0.83$ & 3.25 & 0.001 & $\begin{array}{l}\mathrm{Tau}^{2}=0.08, \text { chi }^{2}=17.65 \\
\mathrm{df}^{\mathrm{d}}=9(P=0.04) \\
I^{2}=49 \%\end{array}$ \\
\hline
\end{tabular}

even more necessary for malnourished children, as they already have a zinc deficiency, which predisposes them to diarrhea and worsens it (50). WHO recommends zinc supplementation for children in developing countries who have acute or persistent diarrhea at a dose of $10 \mathrm{mg}$ in infants less than 6 months old and $20 \mathrm{mg}$ in older infants daily for $10-14$ days $(51,52)$.

Obtaining homogeneous results in the entire population was not possible. The 
FIGURE 4. Incidence of vomiting in included studies ${ }^{a}$

\begin{tabular}{|c|c|c|c|c|c|c|c|c|c|}
\hline \multirow{2}{*}{$\begin{array}{l}\text { Study or Subgroup } \\
\text { 1.6.1 Age }>6 \text { months }\end{array}$} & Zinc & Total & \multicolumn{2}{|c|}{ Control } & \multicolumn{3}{|c|}{ Risk Ratio } & \multicolumn{2}{|c|}{$\begin{array}{c}\text { Risk Ratio } \\
\text { M-H, Random, } 95 \% \mathrm{Cl}\end{array}$} \\
\hline & & & & & & & & & \\
\hline Sachdev 1988 & 0 & 25 & 0 & 25 & & Not estimable & 1988 & & \\
\hline Sazawal 1995 & 2 & 456 & 2 & 481 & $0.8 \%$ & $1.05[0.15,7.46]$ & 1995 & & \\
\hline $\begin{array}{l}\text { Strand } 2002 \\
\text { Subtotal }(95 \% \mathrm{Cl})\end{array}$ & 145 & $\begin{array}{l}442 \\
923\end{array}$ & 85 & $\begin{array}{l}449 \\
955\end{array}$ & $\begin{array}{l}55.0 \% \\
55.8 \%\end{array}$ & $\begin{array}{l}1.73[1.37,2.19] \\
1.72[1.36,2.17]\end{array}$ & 2002 & & \\
\hline \multicolumn{10}{|c|}{$\begin{array}{l}\text { Heterogeneity: } \mathrm{Tau}^{2}=0.00 ; \mathrm{Chi}^{2}=0.24, \mathrm{df}=1(P=0.62) ; l^{2}=0 \% \\
\text { Test for overall effect: } Z=4.59(P<0.00001)\end{array}$} \\
\hline \multicolumn{10}{|c|}{ 1.6.2 Age $<$ and $>6$ months } \\
\hline Polat 2003 low Zn level & 8 & 40 & 2 & 36 & $1.4 \%$ & $3.60[0.82,15.86]$ & 2003 & & \\
\hline Polat 2003 normal Zn lev & 11 & 52 & 3 & 54 & $2.0 \%$ & $3.81[1.13,12.88]$ & 2003 & & \\
\hline $\begin{array}{l}\text { Larson } 2005 \\
\text { Subtotal }(95 \% \mathrm{Cl})\end{array}$ & 139 & $\begin{array}{l}534 \\
626\end{array}$ & 64 & $\begin{array}{l}533 \\
623\end{array}$ & $\begin{array}{l}40.9 \% \\
44.2 \%\end{array}$ & $\begin{array}{l}2.17[1.65,2.84] \\
2.26[1.74,2.93]\end{array}$ & 2005 & & \\
\hline \multicolumn{10}{|c|}{$\begin{array}{l}\text { Heterogeneity: } \operatorname{Tau}^{2}=0.00 ; \mathrm{Chi}^{2}=1.18, \mathrm{df}=2(P=0.55) ; p^{2}=0 \% \\
\text { Test for overall effect: } Z=6.14(P<0.00001)\end{array}$} \\
\hline Total $(95 \% \mathrm{Cl})$ & & 1549 & & 1578 & $100.0 \%$ & $1.94[1.63,2.31]$ & & & $\boldsymbol{P}$ \\
\hline Total events & 305 & & 156 & & & & & & \\
\hline $\begin{array}{l}\text { Heterogeneity: } \mathrm{Tau}^{2}=0.00 \\
\text { Test for overall effect: } Z=\end{array}$ & $\begin{array}{l}\mathrm{Chi}^{2}=3.7 \\
51(P<0 \\
\left(P<\mathrm{C}^{2}=\right.\end{array}$ & $\begin{array}{l}79, \mathrm{df}= \\
.00001) \\
235 . \mathrm{df}\end{array}$ & $4(P=0.4$ & 44); $/^{2}=$ & $=0 \%$ & & & $\begin{array}{cccc}1 & 1 & 1 & 1 \\
0.1 & 0.2 & 0.5 & 1 \\
& \text { Favors Zinc }\end{array}$ & 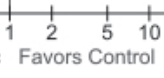 \\
\hline
\end{tabular}

a M-H: Mantel-Haenszel, Cl: confidence interval, df: degrees of freedom.

FIGURE 5. Diarrhea duration (hours) in malnourished children ${ }^{a}$

\begin{tabular}{|c|c|c|c|c|c|c|c|c|c|c|c|c|}
\hline \multirow[b]{2}{*}{ Study or Subgroup } & \multicolumn{3}{|c|}{ Zinc } & \multicolumn{3}{|c|}{ Control } & \multicolumn{3}{|c|}{ Mean Difference } & \multirow{2}{*}{\multicolumn{3}{|c|}{$\begin{array}{l}\text { Mean Difference } \\
\text { IV, Random, } 95 \% \mathrm{Cl}\end{array}$}} \\
\hline & Mean & SD & Total & Mean & SD & Total & Weight & IV, Random, $95 \% \mathrm{Cl}$ & Year & & & \\
\hline \multicolumn{13}{|l|}{ 1.1.1 age $>6$ months } \\
\hline $\begin{array}{l}\text { Sachdev } 1988 \\
\text { Subtotal }(95 \% \mathrm{Cl})\end{array}$ & 65.5 & 27.2 & $\begin{array}{l}11 \\
11\end{array}$ & 97.1 & 43.7 & $\begin{array}{l}14 \\
14\end{array}$ & $\begin{array}{l}3.7 \% \\
3.7 \%\end{array}$ & $\begin{array}{l}-31.60[-59.57,-3.63] \\
-31.60[-59.57,-3.63]\end{array}$ & 1988 & & & \\
\hline \multicolumn{13}{|c|}{$\begin{array}{l}\text { Heterogeneity: Not applicable } \\
\text { Test for overall effect: } Z=2.21(P=0.03)\end{array}$} \\
\hline \multicolumn{13}{|c|}{ 1.1.2 age $<$ and $>6$ months } \\
\hline Roy 1997 & 120 & 60 & 37 & 139.2 & 32.7 & 37 & $6.0 \%$ & $-19.20[-41.22,2.82]$ & 1997 & & & \\
\hline Dutta 2000 & 70.4 & 10 & 44 & 103.4 & 17.1 & 36 & $72.6 \%$ & $-33.00[-39.32,-26.68]$ & 2000 & & - & \\
\hline Al-Sonboli 2003 & 26.4 & 16.8 & 21 & 62.4 & 43.2 & 20 & $7.1 \%$ & $-36.00[-56.25,-15.75]$ & 2003 & & & \\
\hline $\begin{array}{l}\text { Polat } 2003 \text { low } \mathrm{Zn} \text { level } \\
\text { Subtotal }(95 \% \mathrm{Cl})\end{array}$ & 105.6 & 31.2 & $\begin{array}{r}40 \\
142\end{array}$ & 146.4 & 40.8 & $\begin{array}{r}36 \\
129\end{array}$ & $\begin{array}{l}10.7 \% \\
96.3 \%\end{array}$ & $\begin{array}{l}-40.80[-57.27,-24.33] \\
-33.23[-38.71,-27.74]\end{array}$ & 2003 & & & \\
\hline \multicolumn{13}{|c|}{$\begin{array}{l}\text { Heterogeneity: } \mathrm{Tau}^{2}=0.00 ; \mathrm{Chi}^{2}=2.45, \mathrm{df}=3(P=0.48) ; I^{2}=0 \% \\
\text { Test for overall effect: } Z=11.87(P<0.00001)\end{array}$} \\
\hline Total $(95 \% \mathrm{Cl})$ & & & 153 & & & 143 & $100.0 \%$ & $-33.17[-38.55,-27.79]$ & & & & \\
\hline \multicolumn{10}{|c|}{$\begin{array}{l}\text { Heterogeneity: } \mathrm{Tau}^{2}=0.00 ; \mathrm{Chi}^{2}=2.46, \mathrm{df}=4(P=0.65) ; l^{2}=0 \% \\
\text { Test for overall effect: } Z=12.08(P<0.00001) \\
\text { Test for subqroup differences: } \mathrm{Chi}^{2}=0.01, \mathrm{df}=1(P=0.91), l^{2}=0 \%\end{array}$} & $\frac{1}{-50}$ & $\begin{array}{c}-25 \\
\text { Favors Zinc }\end{array}$ & Favors Control \\
\hline
\end{tabular}

heterogeneity observed in the included studies was most likely caused by nonstratification of the population by nutritional status, as the analysis showed that the effect of zinc was more pronounced and consistent among stratified studies and studies that included only malnourished children. The heterogeneity observed raises concerns about the external validity of the data $(16,53)$; however, this phenomenon has been an issue in previous systematic reviews, which could not find other plausible explanations for the differences across study results.

No new evidence was found related to children less than 6 months old, thus leaving intact the conclusions drawn in the Cochrane review (7) about the lack of benefit of zinc administration at that age.

Although an evaluation of the mortality rate was not the objective of this study, it can be hypothesized that the use of zinc in conjunction with oral rehydration therapy would contribute to decrease child mortality by reducing the duration of diarrhea, especially in children less than 5 years old. Nevertheless, further studies to evaluate the mortality rate reduction due to the use of zinc in children with diarrhea are required to confirm this hypothesis.

The results summarize the most recent body of literature in the field and support the WHO recommendation to in- clude zinc with oral rehydration therapy for children with diarrhea under the age of 5 years $(1,5)$. This recommendation is supported by cost studies that show zinc is a cost-effective strategy for treating childhood diarrhea $(41,54,55)$. Opposing results were found in a recent evaluation of zinc and copper use in children with diarrhea, but the authors did not consider the entire body of evidence for assessing the effectiveness of zinc (56).

\section{Conclusion}

Oral zinc supplementation significantly decreases the duration of diarrhea and has a greater effect on malnourished 
children. As a public health measure, zinc administration is a good strategy for preventing diarrhea in addition to the use of oral rehydration therapy and is particularly important in countries where zinc deficiency is prevalent. Further research should consider presenting the results according to the nutritional status of the children to provide evidence with greater applicability.

Acknowledgments. The authors thank Paulo Henrique Faria Domingues for helping with text revision.

\section{REFERENCES}

1. Santosham M, Chandran A, Fitzwater S, Fischer-Walker C, Baqui AH, Black R. Progress and barriers for the control of diarrhoeal disease. Lancet. 2010;376(9734):63-7.

2. United Nations Children's Fund, World Health Organization. WHO/UNICEF levels and trends in child mortality. Report 2011: estimates developed by the UN Inter-agency Group for Child Mortality Estimation. New York: UNICEF; 2010. Available from: http:/ / www.childinfo.org/files/Child_Mortality_Report_2011.pdf Accessed 11 January 2013.

3. Wardlaw T, Salama P, Brocklehurst C, Chopra M, Mason E. Diarrhoea: why children are still dying and what can be done. Lancet. 2010;375:870-2.

4. Walker CLF, Black RE. Zinc for the treatment of diarrhoea: effect on diarrhoea morbidity, mortality and incidence of future episodes. Int J Epidemiol. 2010;39:63-9.

5. United Nations Children's Fund, World Health Organization. Diarrhoea: why children are still dying and what can be done. New York: UNICEF, WHO; 2009. Available from: http://whqlibdoc.who.int/publica tions/2009/9789241598415_eng.pdf Accessed 11 Janury 2013.

6. Jones G, Steketee RW, Black RE, Bhutta ZA, Morris SS, Bellagio Child Survival Study G. How many child deaths can we prevent this year? Lancet. 2003;362(9377):65-71.

7. Lazzerini M, Ronfani L. Oral zinc for treating diarrhoea in children. Cochrane Database Syst Rev. 2012;6:CD005436.

8. Patro B, GolickiD, Szajewska H. Meta-analysis: zinc supplementation for acute gastroenteritis in children. Aliment Pharmacol Ther. 2008;28(6):713-23.

9. Bhutta ZA, Nizami SQ, Isani Z. Zinc supplementation in malnourished children with persistent diarrhea in Pakistan. Pediatrics. 1999;103(4).

10. Haider BA, Bhutta ZA. The effect of therapeutic zinc supplementation among young children with selected infections: a review of the evidence. Food Nutr Bull. 2009;30(1):S41-S59.

11. Lukacik M, Thomas RL, Aranda JV. A metaanalysis of the effects of oral zinc in the treatment of acute and persistent diarrhea. Pediatrics. 2008;121(2):326-36.

12. Naheed A, Walker CLF, Mondal D, Ahmed S, El Arifeen S, Yunus M, et al. Zinc therapy for diarrhoea improves growth among Bangladeshi infants 6 to 11 months of age. J Pediatr Gastroenterol Nutr. 2009;48(1):89-93.

13. Ashworth A, Khanum S, Jackson A, Schofiel C. Guidelines for the inpatient treatment of severely malnourished children. Geneva: WHO; 2003. Available from: http://whqlibdoc. who.int/publications / 2003/9241546093. pdf Accessed 11 January 2013.

14. World Health Organization. Global database on child growth and malnutrition. Geneva: WHO; 2013. Available from: http://www. who.int/nutgrowthdb/about/introduction/en/index5.html Accessed 11 January 2013.

15. United Nations Children's Fund. Acute malnutrition: classification. New York: UNICEF. Available from: http://www.unicef.org/ nutrition/training/2.3/13.html Accessed 11 January 2013.

16. Higgins JPT, Green S, eds. Cochrane handbook for systematic seviews of interventions. Version 5.1.0. Oxford: The Cochrane Collaboration; 2011. Available from: http://www. cochrane-handbook.org Accessed 11 january 2013.

17. Arora R, Kulshreshtha S, Mohan G, Singh M, Sharma P. Estimation of serum zinc and copper in children with acute diarrhea. Biol Trace Elem Res. 2006;114(1-3):121-6.

18. Awasthi S, da Cunha A, Dans LF, El Sayed HF, Gregorio GV, Jain D, et al. Zinc supplementation in acute diarrhea is acceptable, does not interfere with oral rehydration, and reduces the use of other medications: a randomized trial in five countries. J Pediatr Gastroenterol Nutr. 2006;42(3):300-5.

19. Sazawal S, Dhingra U, Deb S, Bhan MK, Menon VP, Black RE. Effect of zinc added to multi-vitamin supplementation containing low-dose vitamin A on plasma retinol level in children-a double-blind randomized, controlled trial. J Health Popul Nutr. 2007;25(1):62-6

20. Roy SK, Hossain MJ, Khatun W, Chakraborty B, Chowdhury S, Begum A, et al. Zinc supplementation in children with cholera in Bangladesh: randomised controlled trial. BMJ. 2008;336(7638):266-8.

21. Chhagan MK, Van den Broeck J, Luabeya KK, Mpontshane N, Tucker KL, Bennish ML. Effect of micronutrient supplementation on diarrhoeal disease among stunted children in rural South Africa. Eur J Clin Nutr. 2009;63(7):850-7.

22. Taneja $\mathrm{S}$, Bhandari $\mathrm{N}$, Rongsen-Chandola $\mathrm{T}$, Mahalanabis D, Fontaine O, Bhan MK, et al. Effect of zinc supplementation on morbidity and growth in hospital-born, low-birth-weight infants. Am J Clin Nutr. 2009;90(2):385-91.

23. Sheikh A, Shamsuzzaman S, Ahmad SM, Nasrin D, Nahar S, Alam MM, et al. Zinc influences innate immune responses in children with enterotoxigenic Escherichia coli-induced

24. Frohna JG. Oral rehydration solution with zinc and prebiotics decreases duration of diarrhea. J Nutr. 2010;140(5):1049-56.
Funding. This research was funded by a public research agency, the Brazilian National Research Council.

Conflicts of interest. The authors have no potential conflicts of interest.

acute diarrhea in children. J Pediatr. 2011; 159(1):166-7.

25. Wadhwa N, Natchu UC, Sommerfelt $\mathrm{H}$, Strand TA, Kapoor V, Saini S, et al. ORS containing zinc does not reduce duration or stool volume of acute diarrhea in hospitalized children. J Pediatr Gastroenterol Nutr. 2011;53(2):161-7.

26. Patel AB, Dibley MJ, Mamtani M, Badhoniya N, Kulkarni H. Therapeutic zinc and copper supplementation in acute diarrhea does not influence short-term morbidity and growth: double-blind randomized controlled trial. Pediatr Infect Dis J. 2013;32(1):91-3.

27. Salvatore $S$, Zuccotti V, Besana R, Diddio D, Luini $C$, Arrigo $S$, et al. Zinc and symbiotic in acute gastroenteritis in Italian children: a pilot study. Dig Liver Dis. 2009;41S(S3):S212-3.

28. Mahlouji K. Effect of zinc supplementation on clinical course of acute diarrhea in patients admitted in Ali Asghar Children Hospital Medical Center Iran University of Medical SciencesTehran Iran. Int J Infect Dis. 2010;14.

29. Sachdev HPS, Mittal NK, Mittal SK, Yadav HS. A controlled trial on utility of oral zinc supplementation in acute dehydrating diarrhea in infants. J Pediatr Gastroenterol Nutr. 1988;7(6):877-81.

30. Sazawal S, Black RE, Bhan MK, Bhandari N, Sinha A, Jalla S. Zinc supplementation in young children with acute diarrhea in India. N Engl J Med. 1995;333(13):839-44.

31. Roy SK, Tomkins AM, Akramuzzaman SM, Behrens RH, Haider R, Mahalanabis D, et al. Randomised controlled trial of zinc supplementation in malnourished Bangladeshi children with acute diarrhoea. Arch Dis Child. 1997;77(3):196-200.

32. Faruque ASG, Mahalanabis D, Haque SS, Fuchs GJ, Habte D. Double-blind, randomized, controlled trial of zinc or vitamin A supplementation in young children with acute diarrhoea. Acta Paediatr. 1999;88(2):154-60.

33. Dutta P, Mitra U, Datta A, Niyogi SK, Dutta S, Manna B, et al. Impact of zinc supplementation in malnourished children with acute watery diarrhoea. J Trop Pediatr. 2000;46(5):25963.

34. Bahl R, Bhandari N, Saksena M, Strand T, Kumar GT, Bhan MK, et al. Efficacy of zincfortified oral rehydration solution in 6-to 35-month-old children with acute diarrhea. J Pediatr. 2002;141(5):677-82.

35. Strand TA, Chandyo RK, Bahl R, Sharma PR, Adhikari RK, Bhandari N, et al. Effectiveness and efficacy of zinc for the treatment of acute diarrhea in young children. Pediatrics. 2002;109(5):898-903.

36. Al-Sonboli N, Gurgel RQ, Shenkin A, Hart CA, Cuevas LE. Zinc supplementation in 
Brazilian children with acute diarrhoea. Ann Trop Paediatr. 2003;23(1):3-8.

37. Polat TB, Uysalol M, Cetinkaya F. Efficacy of zinc supplementation on the severity and duration of diarrhea in malnourished Turkish children. Pediatr Int. 2003;45(5):555-9.

38. Bhatnagar S, Bahl R, Sharma PK, Kumar GT, Saxena SK, Bhan MK. Zinc with oral rehydration therapy reduces stool output and duration of diarrhea in hospitalized children: a randomized controlled trial. J Pediatr Gastroenterol Nutr. 2004;38(1):34-40.

39. Larson CP, Hoque A, Khan AM, Saha UR. Initiation of zinc treatment for acute childhood diarrhoea and risk for vomiting or regurgitation: a randomized, double-blind, placebo-controlled trial. J Health Popul Nutr. 2005;23(4):311-9.

40. Boran P, Tokuc G, Vagas E, Oktem S, Gokduman MK. Impact of zinc supplementation in children with acute diarrhoea in Turkey. Arch Dis Child. 2006;91(4):296-9.

41. Gregorio GV, Dans LF, Cordero CP, Panelo $\mathrm{CA}$. Zinc supplementation reduced cost and duration of acute diarrhea in children. J Clin Epidemiol. 2007;60(6):560-6.

42. Roy SK, Raqib R, Khatun W, Azim T, Chowdhury R, Fuchs GJ, et al. Zinc supplementation in the management of shigellosis in malnourished children in Bangladesh. Eur J Clin Nutr. 2008;62(7):849-55.

43. Patel A, Dibley MJ, Mamtani M, Badhoniya N, Kulkarni H. Zinc and copper supplementation in acute diarrhea in children: a double- blind randomized controlled trial. BMC Med. 2009;7.

44. Patro B, Szymański H, Szajewska H. Oral zinc for the treatment of acute gastroenteritis in Polish children: a randomized, double-blind, placebo-controlled trial. J Pediatr. 2010;157(6):984-8.e1.

45. Dalgic N, Sancar M, Bayraktar B, Pullu M, Hasim O. Probiotic, zinc and lactose-free formula in children with rotavirus diarrhea: are they effective? Pediatr Int. 2011;53(5):677-82.

46. Dutta P, Mitra U, Dutta S, Naik TN, Rajendran K, Chatterjee MK. Zinc, vitamin A, and micronutrient supplementation in children with diarrhea: a randomized controlled clinical trial of combination therapy versus monotherapy. J Pediatr. 2011;159(4):633-7.

47. Begg CB, Mazumdar M. Operating characteristics of a rank correlation test for publication bias. Biometrics. 1994;50(4):1088-101.

48. International Monetary Fund. World economic outlook 2009. Washington, D.C.: International Monetary Fund; 2009. Available from: http://www.imf.org/external/ pubs/ft/weo/2009/01/weodata/groups. htm\#oae Accessed 11 January 2013.

49. Kulkarni H, Mamtani M, Patel A. Roles of zinc in the pathophysiology of acute diarrhea. Curr Infect Dis Rep. 2012;14(1):24-32.

50. Berni Canani R, Buccigrossi V, Passariello A. Mechanisms of action of zinc in acute diarrhea. Curr Opin Gastroenterol. 2011;27(1):8-12.

51. United Nations Children's Fund, World Health Organization. WHO/UNICEF joint statement: clinical management of acute diarrhoea. New York: UNICEF, WHO; 2004. Available from: http://whqlibdoc.who. int/hq/2004/WHO_FCH_CAH_04.7.pdf Accessed 11 January 2013.

52. World Health Organization. The treatment of diarrhoea: a manual for physicians and other senior health workers. Geneva: WHO; 2005. Available from: http://apps.who.int/iris/ bitstream/10665/43209/1/9241593180. pdf Accessed 11 January 2013.

53. Borenstein $M$, Hedges LV, Higgins JPT, Rothstein HR. Introduction to meta-analysis. Chichester: Wiley; 2009.

54. Robberstad B, Strand T, Black RE, Sommerfelt $H$. Cost-effectiveness of zinc as adjunct therapy for acute childhood diarrhoea in developing countries. Bull World Health Organ. 2004;82(7):523-31.

55. Baqui AH, Black RE, El Arifeen S, Yunus M, Zaman K, Begum N, et al. Zinc therapy for diarrhoea increased the use of oral rehydration therapy and reduced the use of antibiotics in Bangladeshi children. J Health Popul Nutr. 2004;22(4):440-2.

56. Patel AB, Badhoniya N, Dibley MJ. Zinc and copper supplementation are not cost-effective interventions in the treatment of acute diarrhea. J Clin Epidemiol. 2013;66(1):52-61

Manuscript received on 25 September 2012. Revised version accepted for publication on 25 February 2013.

RESUMEN Objetivo. Actualizar los datos probatorios disponibles acerca del uso del zinc en el tratamiento de la diarrea infantil y evaluar su efecto en la población malnutrida, un subgrupo no plenamente estudiado en análisis anteriores.

Administración de suplementos de zinc para tratar la diarrea infantil: revisión sistemática y metanálisis

Métodos. Se llevó a cabo una revisión sistemática de ensayos clínicos aleatorizados que evaluaban a niños de hasta 5 años de edad con diarrea aguda y a los que se les había administrado suplementos de zinc. A los controles se les había administrado un placebo o terapia de rehidratación oral. Después de efectuar búsquedas en las principales bases de datos, sin restricciones en cuanto a idiomas, dos revisores independientes seleccionaron los estudios idóneos, extrajeron los datos y evaluaron el riesgo de sesgo de los estudios incluidos. El metanálisis se efectuó mediante el modelo de efectos aleatorios de Mantel-Haenszel o de la varizanza inversa.

Resultados. En la revisión se incluyeron 18 estudios de los 1041 recuperados ( $n=7314$ niños). El zinc tuvo un efecto beneficioso en la reducción de la duración de la diarrea en horas (diferencia media $[D M]=-20,12$, intervalo de confianza del $95 \%$ [IC] de $-29,15$ a $\left.-11,09, I^{2}=91 \%\right)$. Este efecto fue más intenso en niños malnutridos $\left(\mathrm{DM}=-33,17\right.$, IC del 95\% = de $\left.-33,55 \mathrm{a}-27,79, I^{2}=0 \%\right)$. La prevalencia de diarrea en los días 3, 5 y 7 fue inferior en el grupo tratado con zinc. La incidencia de vómitos fue significativamente mayor en el grupo tratado con zinc que en el grupo de referencia. Los ensayos aleatorizados controlados que fueron incluidos mostraron un bajo riesgo de sesgo en la mayoría de los dominios evaluados.

Conclusiones. La administración oral de suplementos de zinc reduce significativamente la duración de la diarrea y tiene un efecto aún mayor en niños malnutridos. La administración de suplementos de zinc parece ser una estrategia de salud pública apropiada, principalmente en las zonas afectadas por carencias endémicas.

Palabras clave Zinc; diarrea; niño; desnutrición; revisión. 